\title{
A Generalized Version of the McMullen Domain
}

\author{
Paul Blanchard \\ Robert L. Devaney \\ Antonio Garijo *† \\ Elizabeth D. Russell ${ }^{\ddagger}$ \\ Boston University \\ Boston, MA 02215 USA
}

November 19, 2007

\begin{abstract}
We study the family of complex maps given by $F_{\lambda}(z)=z^{n}+$ $\lambda / z^{n}+c$ where $n \geq 3$ is an integer, $\lambda$ is an arbitrarily small complex parameter, and $c$ is chosen to be the center of a hyperbolic component of the corresponding Multibrot set. We focus on the structure of the Julia set for a map of this form generalizing a result of McMullen. We prove that it consists of a countable collection of Cantor sets of closed curves and an uncountable number of point components.
\end{abstract}

${ }^{*}$ Dep. d'Eng. Informàtica i Matemàtiques, Universitat Rovira i Virgili, Av. Països Catalans, 26, 43007 Tarragona, Spain

${ }^{\dagger}$ The third author would like to thank to Department of Mathematics at Boston University for their hospitality while this work was in progress. The third author was supported by MTM2005-02139/Consolider (including a FEDER contribution) and CIRIT 2005 SGR01028.

${ }_{\ddagger}^{\ddagger}$ Please address all correspondence to Elizabeth D. Russell, Department of Mathematics, Boston University, 111 Cummington Street, Boston MA 02215, or e-mail erussell@bu.edu. 


\section{Introduction}

In this paper we consider complex rational maps of the form

$$
F_{\lambda}(z)=z^{n}+\frac{\lambda}{z^{n}}+c
$$

where $\lambda, c \in \mathbb{C}$ are parameters. For this family of maps, we fix $c$ to be the center of a hyperbolic component of the corresponding Multibrot set, i.e., a parameter such that, for the map

$$
F_{0}(z)=z^{n}+c
$$

0 lies in a superattracting cycle. For reasons explained below, we shall assume that $n \geq 3$ and that $c$ is chosen so that this superattracting cycle has period $k>1$. The maps $F_{\lambda}$ are obtained by replacing the only finite critical point of $F_{0}$ with a pole of order $n$. For these maps, $\infty$ is always a superattracting fixed point, so there is an immediate basin of $\infty$ that we denote by $B_{\lambda}$. Since 0 is the only pole of $F_{\lambda}$ when $\lambda \neq 0$, there is a preimage of $B_{\lambda}$ that contains 0 . If this preimage of $B_{\lambda}$ is disjoint from $B_{\lambda}$, this set is called the trap door and denoted $T_{\lambda}$. In this paper, we shall only consider cases where $B_{\lambda}$ and $T_{\lambda}$ are disjoint, which always happens when $|\lambda|$ is sufficiently small.

For the special case $c=0$, the origin is a superattracting fixed point of $F_{0}$. The maps $F_{\lambda}$ in this case have been extensively studied; see [Devaney et al., to appear], [Devaney et al., 2005], [Devaney \& Look, 2006]. When $\lambda=0$, the Julia set for this family is the unit circle. When $\lambda \neq 0$, there are $2 n$ "free" critical points for $F_{\lambda}$, i.e., critical points not equal to 0 or $\infty$. Each of the free critical points maps to one of two critical values. When $n$ is even, these critical values then map to the same point so there is really only one free critical orbit in this case. When $n$ is odd, the two critical values map to different points, but these points and their subsequent orbits are always symmetric under $z \mapsto-z$, so again, up to this symmetry, there is only one 
critical orbit. Similarly, for the family $z^{n}+c$, there is only one critical orbit. For $z^{n}+c$, either the critical orbit escapes to $\infty$ or it does not. In the former case, it is well known that the Julia set is a Cantor set, whereas, in the latter case, the Julia set is a connected set. For the maps $F_{\lambda}$, it turns out that there are three possible ways that the critical orbit can escape to $\infty$, so the situation is more complicated. The following is proven in [Devaney et al., 2005]:

Theorem. The Escape Trichotomy. Suppose $n \geq 3$. Suppose that the orbits of the free critical points of $F_{\lambda}(z)=z^{n}+\lambda / z^{n}$ tend to $\infty$.

1. If the critical values lie in $B_{\lambda}$, then $J\left(F_{\lambda}\right)$ is a Cantor set and $F_{\lambda} \mid J\left(F_{\lambda}\right)$ is conjugate to a one-sided shift on $2 n$ symbols.

2. If the critical values lie in $T_{\lambda}$, then $J\left(F_{\lambda}\right)$ is a Cantor set of concentric simple closed curves (quasicircles).

3. If the critical values eventually map into $T_{\lambda}$, then $J\left(F_{\lambda}\right)$ is a Sierpinski curve.

We remark that case 2 of this result was proven by McMullen [1988]. Moreover, this case does not occur when $n=2$. This is the reason why we assume that $n \geq 3$.

In the above result, a Sierpinski curve is a planar set that is homeomorphic to the Sierpinski carpet fractal. It is known that any planar set that is compact, connected, locally connected, nowhere dense, and has the property that any two complementary domains are bounded by disjoint simple closed curves is homeomorphic to the carpet [Whyburn, 1958]. It is also known that a Sierpinski curve is a universal plane continuum since there is a homeomorphic copy of any planar, one-dimensional, compact, connected set contained in a Sierpinski curve. 
In this paper we will be especially interested in the second case of the Escape Trichotomy. The set of $\lambda$ values such that the Julia set of $z^{n}+\lambda / z^{n}$ is a Cantor set of simple closed curves is called the McMullen domain. It is known that the McMullen domain is an open disk in the parameter plane that is punctured at the origin and bounded by a simple closed curve [Devaney $\&$ Marotta, 2007]. For $\lambda$-values in the McMullen domain, it is also known that the boundary of $B_{\lambda}$, denoted by $\partial B_{\lambda}$, is a simple closed curve, just as in the case where $\lambda=0$. But when $\lambda \neq 0$, the Julia set explodes and becomes a Cantor set of simple closed curves. When we say that the Julia set is a Cantor set of simple closed curves, we mean that this set is homeomorphic to the cross-product of a Cantor set with the unit circle having the property that all of the closed curves in this set are concentric.

Our goal in this paper is to show that a somewhat different phenomenon occurs when $c$ is chosen at the center of a hyperbolic component of the Multibrot set with period $k>1$. In this case, it is known that the interior of $K\left(F_{0}\right)$, the filled Julia set, now consists of infinitely many disjoint open disks, so $J\left(F_{0}\right)$ is a much more intricate set than in the case $c=0$. As is well known, $J\left(F_{0}\right)$ is the boundary of the basin of attraction of $\infty$. We shall show that, for $|\lambda|$ sufficiently small, $\partial B_{\lambda}$ is still homeomorphic to $J\left(F_{0}\right)$ and $F_{\lambda} \mid \partial B_{\lambda}$ is conjugate to $F_{0}$ on $J\left(F_{0}\right)$. As in the case $c=0$, there is again much more to the Julia set when $\lambda$ becomes nonzero. We shall show that, again for $|\lambda|$ sufficiently small, the $k^{\text {th }}$ iterates of the critical points all lie in the trap door. This will imply that the Julia set now contains countably many Cantor sets of simple closed curves; indeed, each of the infinitely many interior components of the complement of $B_{\lambda}$ now contains a Cantor set of simple closed curves. However, the simple closed curves corresponding to endpoints in the Cantor set are quite different: each of them has infinitely many additional "decorations" attached. 
There is also a great deal more to these Julia sets besides this countable collection of Cantor sets of closed curves and the boundary of the basin of $\infty$. In particular, most of the the Cantor sets of closed curves do not contain any periodic points. As is well known, the repelling periodic points of $F_{\lambda}$ are dense in $J\left(F_{\lambda}\right)$, so these periodic points must lie elsewhere in the Julia set. To describe these other points in the Julia set, we will assign an itinerary to these other points in the Julia set. This itinerary will be an infinite sequence of integers that describes how the orbit of this point moves through the interior components of the complement of $B_{\lambda}$. We will show that the set of points whose itinerary ends in certain sequences forms a Cantor set, and these points form the remainder of the Julia set. Finally, we will prove that each of the points in these Cantor sets are point components of the Julia set.

Thus our main goal in this paper is to prove the following:

Theorem. Let $n \geq 3$. Consider $z^{n}+\lambda / z^{n}+c$, where $c$ lies at the center of a hyperbolic component in the Multibrot set of period $k>1$ for $z^{n}+c$. When $|\lambda|$ is sufficiently small, the Julia set contains

1. countably many preimages of the boundary of $B_{\lambda}$;

2. a countable collection of Cantor sets of closed curves;

3. and an uncountable number of point components.

We remark that the "unburied" curves in the Cantor set of simple closed curves are the ones that come with the "decorations" attached; these curves are actually preimages of $\partial B_{\lambda}$.

To illustrate this result, in Fig. 1, we display the Julia set of $z^{3}+\lambda / z^{3}-i$ when $\lambda=0.0001$ and of $z^{3}-i$, the unperturbed map. The outer boundary of the Julia set of $z^{3}+\lambda / z^{3}-i$ is a homeomorphic copy of the Julia set 

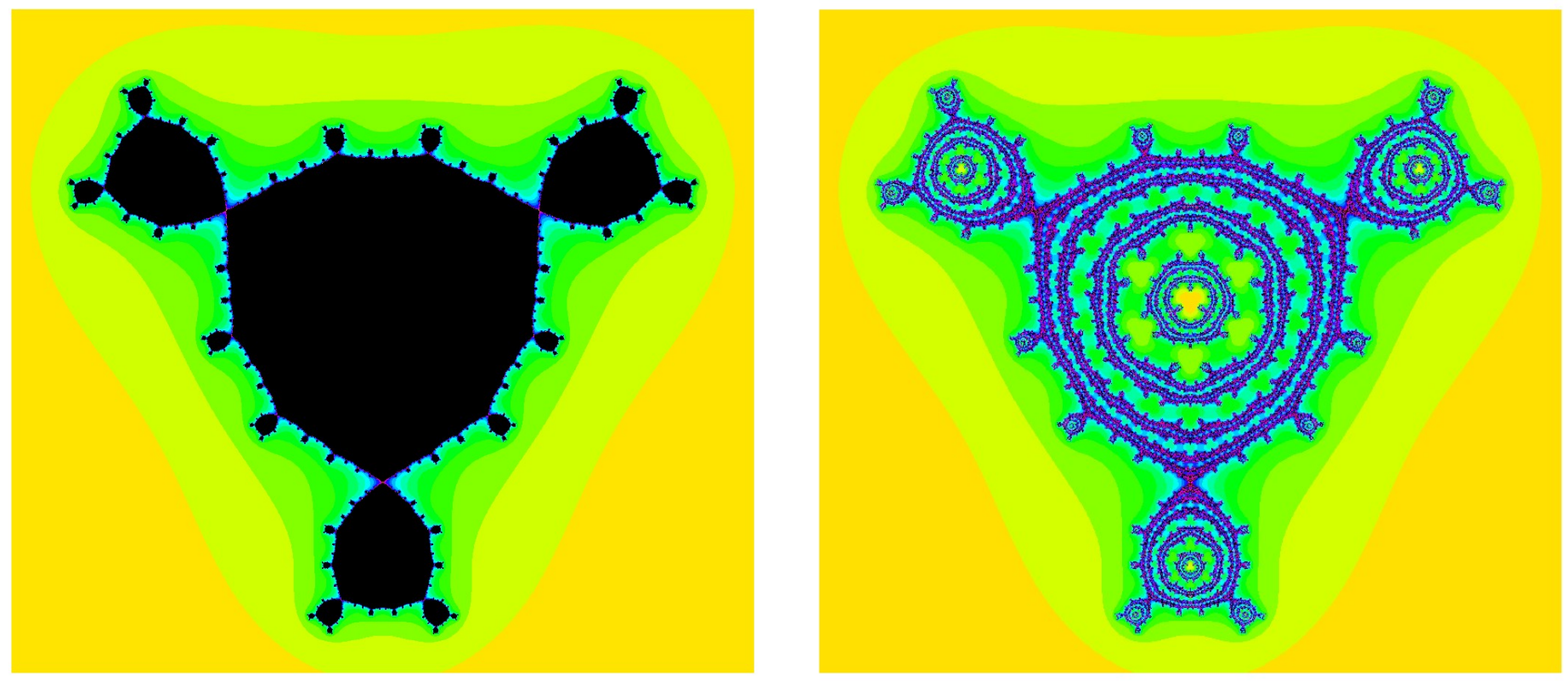

Figure 1: The Julia sets for $z^{3}-i$ (left) and $z^{3}+\lambda / z^{3}-i$ when $\lambda=0.0001$ (right). The critical point in the unperturbed map, $z^{3}-i$, is in a superattracting 2-cycle. Note the explosion that occurs when $\lambda$ becomes nonzero.

of $z^{3}-i$. Note that 0 lies on a 2-cycle for $z^{3}-i$, so $-i$ is the center of a hyperbolic component of period two for $z^{3}+c$. The central yellow region in the picture to the right is the trap door. Note that this region is surrounded by a collection of open annuli whose boundaries are not simple closed curves, but rather have a structure similar to the attachments in the Julia set of $z^{3}-i$; these are the decorated curves. Figure 2 shows magnifications of several regions in this Julia set. In Fig. 3, we display another cubic Julia set and a perturbation to it; when $\lambda=0$, there is an attracting four cycle. Figure 4 shows a magnification of the trap door of this Julia set.

We also remark that the situation that occurs when $n=2$ is dramatically different. In this case, there are no Cantor sets of circles. Rather, the interior regions of the Fatou set of $F_{0}$ are often filled with collections of Sierpinski curves. See [Devaney et al., to appear]. 

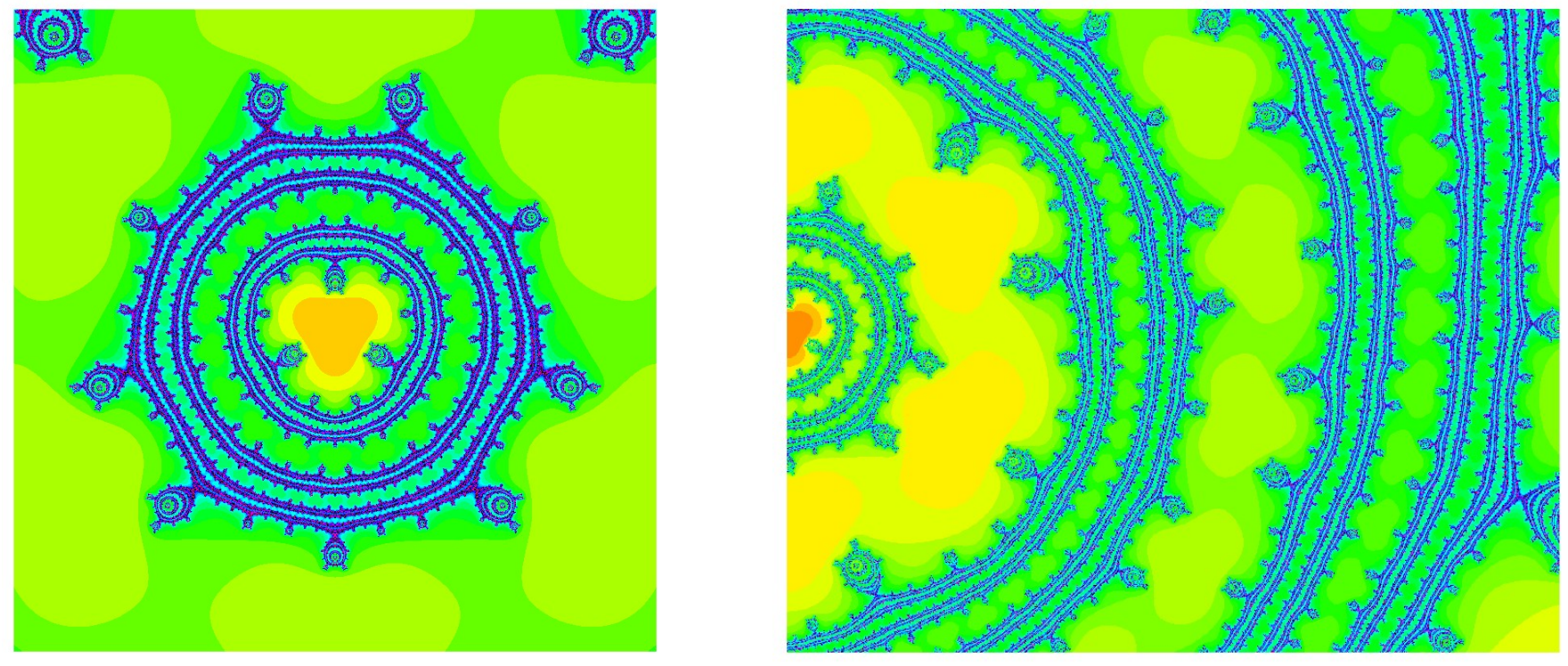

Figure 2: Magnifications of Fig. 1: the trap door (left) and a section of the Cantor set of closed curves (right). Note the attachments to the accessible circles.

\section{Preliminaries}

Let $c$ be the center of a hyperbolic component of the Multibrot set with period greater than one. Let

$$
F_{\lambda}(z)=z^{n}+\frac{\lambda}{z^{n}}+c
$$

where $\lambda, c \in \mathbb{C}$ and $n \geq 3$. When $\lambda \neq 0$, maps of this form have critical points at $0, \infty$ and the $2 n$ points located symmetrically about the origin at $\lambda^{1 / 2 n}$. Since 0 is mapped to $\infty$ which is superattracting fixed point, we call the remaining $2 n$ critical points the free critical points. The $2 n$ critical points map to $v_{\lambda}=c \pm 2 \lambda^{1 / 2}$, the two critical values for $F_{\lambda}$. There are thus only two critical orbits since, after one iteration, each of the critical points is mapped to one of these two points. This is one difference between our family and the 

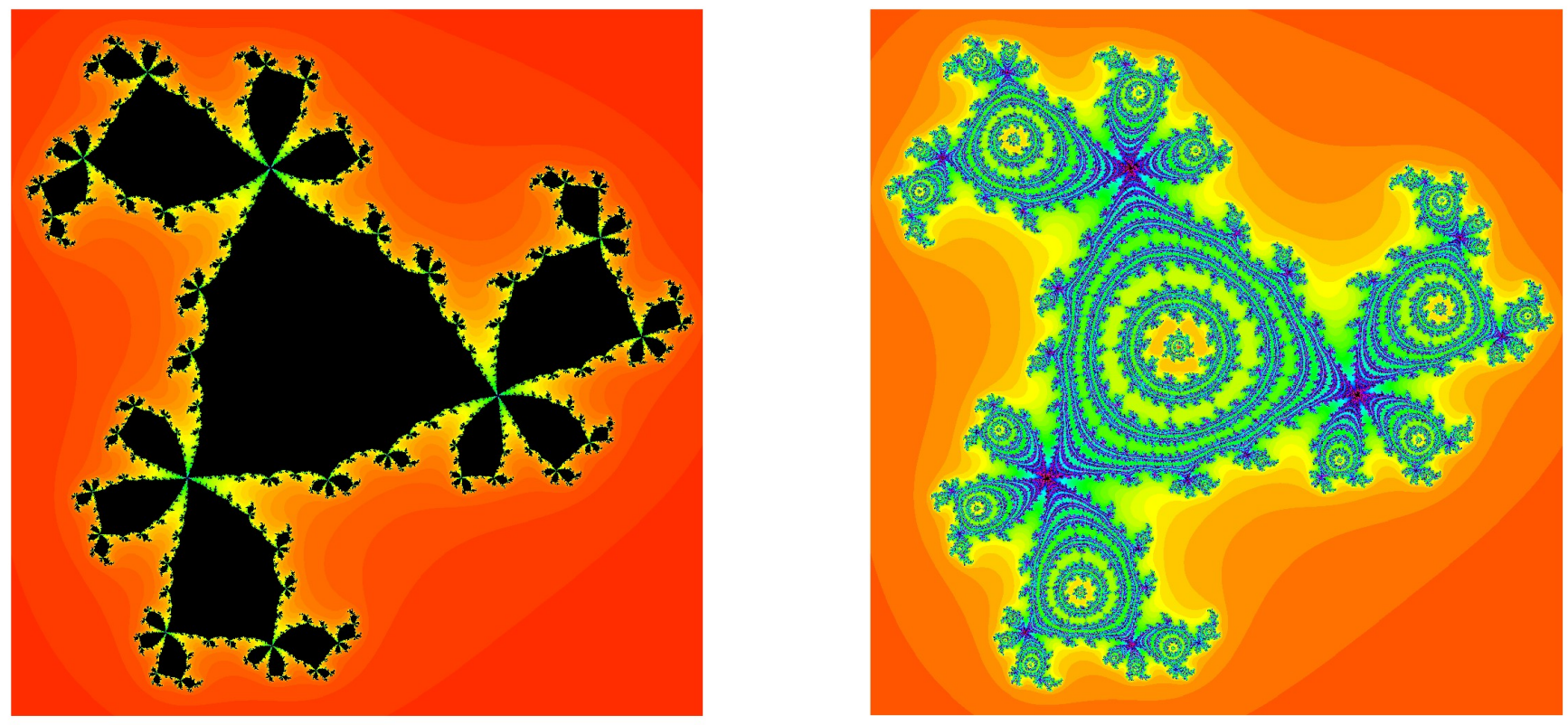

Figure 3: The Julia set for $z^{3}+c$ (left) and $z^{3}+\lambda / z^{3}+c$ (right) when $c=-0.58384-0.27022 i$ and $\lambda=-0.0000001$. The critical point of the unperturbed map, $z^{3}-0.58384-0.27022 i$, is in a superattracting 4-cycle. 


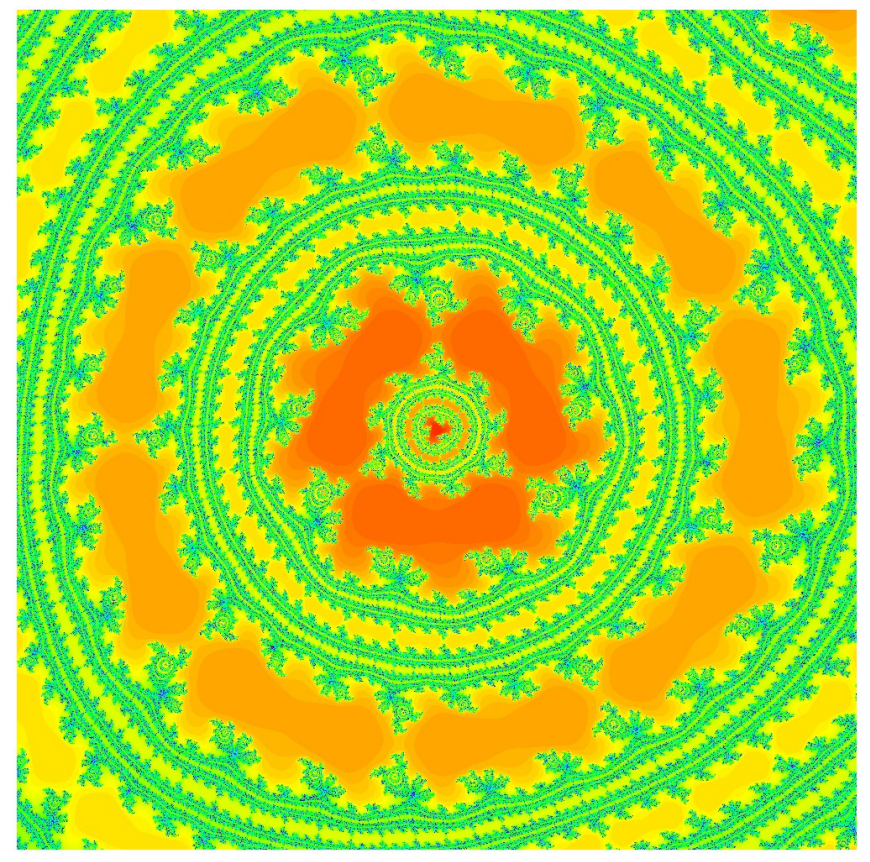

Figure 4: A magnification of the trap door of the Julia set for $z^{3}+\lambda / z^{3}+c$ when $c=-0.58384-0.27022 i$ and $\lambda=-0.0000001$.

case $c=0$; when $c=0$ there is only one free critical orbit.

It is easily verified that the circle of radius $\left|\lambda^{1 / 2 n}\right|$ that connects the $2 n$ critical points is mapped $2 n$ times over the line segment connecting the two critical values, $c \pm 2 \lambda^{1 / 2}$. We call the circle of radius $\left|\lambda^{1 / 2 n}\right|$ the critical circle and the line segment connecting the two critical values the critical segment. It is also easily verified that any circle centered at the origin with the exception of the critical circle is mapped $n$-to- 1 onto an ellipse whose two foci are the critical values. Thus each component of the complement of the critical circle in $\mathbb{C}$ is mapped as an $n$-to- 1 covering of the complement of the critical segment.

Since $\infty$ is a superattracting fixed point, it has an immediate basin of attraction denoted by $B_{\lambda}$. When $\lambda \neq 0$, there is a neighborhood of 0 that maps to $B_{\lambda}$. If this neighborhood is disjoint from $B_{\lambda}$, we call the component of the basin of infinity containing the origin the trap door and denote it by 
$T_{\lambda}$. In this paper, we will consider only maps of this type, i.e., those maps with $|\lambda|$ chosen sufficiently small so that $T_{\lambda}$ and $B_{\lambda}$ are disjoint.

The Julia set of $F_{\lambda}$ is the set on which the family of iterates of $F_{\lambda}$ fails to be normal in the sense of Montel. It is known that this set is equivalently the closure of the set of repelling periodic points of $F_{\lambda}$. The complement of the Julia set is called the Fatou set.

When $\lambda=0$, the critical point 0 lies in a superattracting cycle of period $k$ given by $0=c_{0}, c=c_{1}, c_{2}, \cdots, c_{k-1}, c_{k}=0$. Let $\mathcal{O}_{j}$ denote the immediate basin of attraction of $c_{j}$ under $F_{0}^{k}$. So $F_{0}$ maps $\mathcal{O}_{0} n$-to-one onto $\mathcal{O}_{1}$, while $F_{0}$ maps each other $\mathcal{O}_{j}$ one-to-one onto $\mathcal{O}_{j+1}$. It follows that $F_{0}^{k}$ is conformally conjugate to $z \mapsto z^{n}$ on each $\mathcal{O}_{j}$.

\section{The Boundary of the Basin of Infinity}

In this section we prove that, if $|\lambda|$ is sufficiently small, the boundary of $B_{\lambda}, \partial B_{\lambda}$, is homeomorphic to $J\left(F_{0}\right)$. As is well known, there is a Böttcher coordinate $\varphi_{\lambda}$ defined on a neighborhood of $\infty$ in $B_{\lambda}$ that conjugates $F_{\lambda}$ to $z \mapsto z^{n}$ in a neighborhood of $\infty$. If none of the free critical points lie in $B_{\lambda}$, then, again as is well known, we may extend the definition of $\varphi_{\lambda}$ so that it takes the entire immediate basin univalently onto $\mathbb{C}-\overline{\mathbb{D}}$ and hence conjugates $F_{\lambda}$ to $z \mapsto z^{n}$ on all of $B_{\lambda}$. The following proposition shows that this occurs.

Proposition. If $|\lambda|$ is sufficiently small, then $T_{\lambda}$ is disjoint from $B_{\lambda}$, so $0 \notin B_{\lambda}$. Also, none of the free critical points lie in $B_{\lambda}$.

Proof: Recall that $\mathcal{O}_{0}$ is the component of the interior of $J\left(F_{0}\right)$ that contains 0 . Let $\nu_{0}$ denote the boundary of $\mathcal{O}_{0}$. We have that $F_{0}^{k}$ preserves $\nu_{0}$ and is hyperbolic on this set since the critical orbit is periodic and hence bounded away from this set. Therefore there is an open annulus containing $\nu_{0}$ that is mapped by $F_{0}^{k}$ as a covering over a larger annulus. For $|\lambda|$ sufficiently small, 
$F_{\lambda}$ maps this annulus in a similar fashion completely over itself. Hence $B_{\lambda}$ cannot extend into the disk that is the bounded component of the complement of this annulus. The critical points are given by $\lambda^{1 / 2 n}$, so this annulus may be chosen so that all of the critical points and 0 lie in this internal disk. Hence they do not lie in $B_{\lambda}$.

Next we prove that $\partial B_{\lambda}$ is homeomorphic to $J\left(F_{0}\right)$ using holomorphic motions. To do this, we first define a holomorphic motion on $B_{\lambda}$ that is parameterized by $\lambda$ for $|\lambda|$ sufficiently small. Then we apply the $\Lambda$-lemma of Manẽ, Sad, and Sullivan [1983] to extend this motion to $\overline{B_{\lambda}}$. This will show that $\partial B_{\lambda}$ is homeomorphic to $\partial B_{0}=J\left(F_{0}\right)$.

Recall the definition of a holomorphic motion:

Definition. Let $X \subset \mathbb{C}$. A map $H: X \times \mathbb{D} \rightarrow \overline{\mathbb{C}}$ given by $(z, \lambda) \rightarrow H(z, \lambda)$ is a holomorphic motion of $X$ parameterized by $\lambda$ if

1. $H(z, 0)=z$ for all $z \in X$;

2. $H(\cdot, \lambda): X \rightarrow \overline{\mathbb{C}}$ is injective for all $z \in X$;

3. $H(z, \cdot): \mathbb{D} \rightarrow \overline{\mathbb{C}}$ is holomorphic for all $z \in X$.

Proposition (The $\Lambda$-Lemma.) Let $H: X \times \mathbb{D} \rightarrow \overline{\mathbb{C}}$ be a holomorphic motion. Then $H$ is continuous and it extends to a unique holomorphic motion $\bar{H}: \bar{X} \times \overline{\mathbb{C}}$, where $\bar{X}$ is the closure of $X$.

As shown above, we may choose $\delta>0$ so that the Böttcher coordinate is defined on all of $B_{\lambda}$ for all $\lambda$ with $|\lambda|<\delta$. If $\mu \in \mathbb{D}$, we have that $\varphi_{\delta \mu}$ is defined on all of $B_{\delta \mu}$. So we define $H: B_{0} \times \mathbb{D} \rightarrow \overline{\mathbb{C}}$ by $H(z, \mu)=\varphi_{\delta \mu}^{-1} \circ \varphi_{0}(z)$. We claim that $H$ is a holomorphic motion. First the identity condition is easily satisfied since $\varphi_{0}^{-1} \circ \varphi_{0}(z)=z . H$ is injective for fixed $\mu$ since $\varphi_{\delta \mu}$ is 
injective. Third, since $H$ is a composition of functions that are holomorphic in $\mu, H$ must also be holomorphic in $\mu$. Thus, $H$ is a holomorphic motion. Applying the $\Lambda$-lemma, we obtain a new map, $\bar{H}: \bar{B}_{0} \times \mathbb{D} \rightarrow \overline{\mathbb{C}}$. It follows that $B_{\delta \mu}$ is the continuous image of $J\left(F_{0}\right)$ under $\bar{H}$. Interchanging 0 and $\delta \mu$, we have that $\bar{H}$ is a homeomorphism. Thus we have shown that the boundary of the basin of infinity of $F_{\lambda}=F_{\delta \mu}$ is homeomorphic to the Julia set of $F_{0}$.

\section{Cantor Sets of Simple Closed Curves}

Our goal in this section is to prove the existence of a countable collection of Cantor sets of simple closed curves in $J\left(F_{\lambda}\right)$. Recall that when $\lambda=0$, the origin lies on a superattracting $k$-cycle. We restrict in this section to values of $|\lambda|$ small enough so that $B_{\lambda}$ is homeomorphic to $J\left(F_{0}\right)$. It follows that the preimage of $B_{\lambda}$, namely $T_{\lambda}$, that contains 0 is disjoint from $B_{\lambda}$. We will first show that, for these $\lambda$-values, the $k^{\text {th }}$ iterate of $c_{\lambda}$ lies in $T_{\lambda}$ so the $(k+1)^{\text {st }}$ iterate of $c_{\lambda}$ lies in $B_{\lambda}$.

First observe the following for $|\lambda|$ small. (We consider the critical value $v_{\lambda}$ to be given by $c+2 \lambda^{1 / 2}$; the same argument can be used for the other critical value.)

$$
\begin{aligned}
c_{\lambda} & =\lambda^{1 / 2 n} \\
v_{\lambda} & =F_{\lambda}\left(c_{\lambda}\right)=c+2 \sqrt{\lambda}=F_{0}(0)+2 \sqrt{\lambda} \\
F_{\lambda}^{2}\left(c_{\lambda}\right) & \approx F_{0}^{2}(0)+F_{0}^{\prime}\left(F_{0}(0)\right) 2 \sqrt{\lambda} \\
F_{\lambda}^{3}\left(c_{\lambda}\right) & \approx F_{0}^{3}(0)+F_{0}^{\prime}\left(F_{0}^{2}(0)\right) F_{0}^{\prime}\left(F_{0}(0)\right) 2 \sqrt{\lambda} .
\end{aligned}
$$

In general, for $j=1, \cdots, k$, the $j^{\text {th }}$ point on the orbit of $c_{\lambda}$ is:

$$
F_{\lambda}^{j}\left(c_{\lambda}\right) \approx F_{0}^{j}(0)+F_{0}^{\prime}\left(F_{0}^{j-1}(0)\right) F_{0}^{\prime}\left(F_{0}^{j-2}(0)\right) \cdots F_{0}^{\prime}(F(0)) 2 \sqrt{\lambda} .
$$


Consider the $k^{\text {th }}$ iterate of $c_{\lambda}$. Since all of the derivative terms above are nonzero and since $F_{0}^{k}(0)=0$, it follows that $F_{\lambda}^{k}\left(c_{\lambda}\right)$ approaches zero as $\lambda$ does. Then the next iterate is given by

$$
F_{\lambda}^{k+1}\left(c_{\lambda}\right)=\left(F_{\lambda}^{k}\left(c_{\lambda}\right)\right)^{n}+\frac{\lambda}{\left(F_{\lambda}^{k}\left(c_{\lambda}\right)\right)^{n}}+c .
$$

As $\lambda \rightarrow 0$, we have:

$$
\begin{aligned}
\lim _{\lambda \rightarrow 0} F^{k+1}\left(c_{\lambda}\right) & \approx \lim _{\lambda \rightarrow 0} \frac{\lambda}{\left(F_{0}^{k}(0)+F_{0}^{\prime}\left(F_{0}^{k-1}(0)\right) F_{0}^{\prime}\left(F_{0}^{k-2}(0)\right) \cdots F_{0}^{\prime}(F(0)) 2 \sqrt{\lambda}\right)^{n}}+c \\
& =\lim _{\lambda \rightarrow 0} \frac{\lambda}{C \lambda^{n / 2}}+c
\end{aligned}
$$

where $C$ is the nonzero constant

$$
2 F_{0}^{\prime}\left(F_{0}^{k-1}(0)\right) F_{0}^{\prime}\left(F_{0}^{k-2}(0)\right) \cdots F_{0}^{\prime}(F(0)) .
$$

As long as $n \geq 3$, this limit is $\infty$. We have shown:

Proposition. For $n \geq 3$, the $(k+1)^{\text {st }}$ iterates of the critical points all lie in $B_{\lambda}$ for sufficiently small values of $|\lambda|$ so, the $k^{\text {th }}$ iterates of the critical points lie in the trap door.

Recall that, for $\lambda=0$, the function $F_{0}(z)=z^{n}+c$ has a superattracting cycle of period $k$ given by $0=c_{0}, c=c_{1}, \ldots, c_{k-1}$. Recall also that $\mathcal{O}_{j}$ is the immediate basin of attraction of $c_{j}$ under $F_{0}^{k}$. The curve tracing the boundary of each of the $\mathcal{O}_{j}$ is denoted by $\nu_{j}$. Each of the $\mathcal{O}_{j}$ is a simply connected Fatou component for the map $F_{0}$. These components of the Fatou set behave in the following way: $\mathcal{O}_{0}$ is mapped $n$-to-one over $\mathcal{O}_{1}$ by $F_{0}$. For $j=1, \ldots, k-2, \mathcal{O}_{j}$ is mapped one-to-one over $\mathcal{O}_{j+1}$ and $\mathcal{O}_{k-1}$ is mapped one-to-one over $\mathcal{O}_{k}=\mathcal{O}_{0}$. The boundary curves of these components map over each other in an analogous manner.

When $\lambda \neq 0$, the $\mathcal{O}_{j}$ persist due to the holomorphic motions argument presented earlier. So for $F_{\lambda}(z)=z^{n}+\lambda / z^{n}+c$ there are analogous regions 
$\mathcal{O}_{0}(\lambda), \ldots, \mathcal{O}_{k-1}(\lambda)$ with the boundaries of these regions defined to be $\nu_{j}(\lambda)$ for $j=0, \ldots, k-1$. The dynamics on $\mathcal{O}_{0}(\lambda)$ is now quite different since, in this case, there is now a pole located at the origin. So the trap door lies inside $\mathcal{O}_{0}(\lambda)$, and so do all of the free critical points. Since the critical values are given by $c \pm 2 \sqrt{\lambda}$, the critical values lie in $\mathcal{O}_{1}(\lambda)$, close to $c$. Hence the boundary of the trap door is mapped as $n$-to-one covering onto the entire set $\partial B_{\lambda}$. We therefore call the boundary of the trap door an $n$-fold inverted copy of the Julia set of $F_{0}$. See Fig. 2.

Let $S_{\lambda}$ be the preimage under $F_{\lambda}$ of the complement of $\mathcal{O}_{1}(\lambda)$ in $\mathcal{O}_{0}(\lambda)$. Note that $S_{\lambda}$ contains the trap door and much more but does not contain the critical points. It follows that the boundary of $S_{\lambda}$ is mapped $n$-to-one onto the boundary of $\mathcal{O}_{1}(\lambda)$ and hence is a simple closed curve that we call $\xi(\lambda)$. So $S_{\lambda}$ is a closed disk bounded by $\xi(\lambda)$. Thus we have that $\xi(\lambda)$ and $\nu_{0}(\lambda)$ are the preimages of $\nu_{1}(\lambda)$. It follows that the preimage of $\mathcal{O}_{1}(\lambda)$ in $\mathcal{O}_{0}(\lambda)$ is an open annulus that is bounded by these two simple closed curves. We call this annulus $\mathcal{A}_{\lambda}$.

Proposition. The preimage under $F_{\lambda}^{k}$ of $S_{\lambda}$ is a closed annulus that divides the annulus $\mathcal{A}_{\lambda}$ into two open subannuli, each of which is mapped univalently onto $\mathcal{A}_{\lambda}$ by $F_{\lambda}^{k}$.

Proof. We have that $S_{\lambda}$ is a closed disk. Suppose that $U=F_{\lambda}^{-k}\left(S_{\lambda}\right)$ has $\ell$ boundary components. Since $F_{\lambda}^{k}$ maps $U$ with degree $2 n$ onto a simply connected set, and there are exactly $2 n$ critical points in $U$, the RiemannHurwitz formula then gives

$$
2-\ell=(2 n)(1-2)+(2 n)=0 .
$$

Thus $U$ has two boundary components forcing it to be either an annulus or two disks. Suppose first that the preimage of the region $S_{\lambda}$ is a pair of disks. 


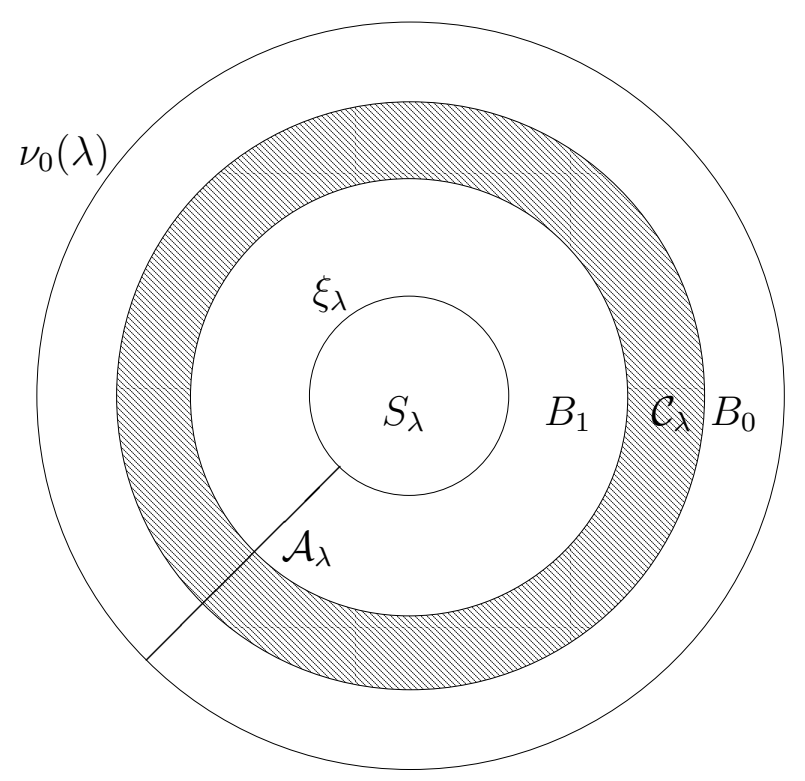

Figure 5: The region $\mathcal{O}_{0}(\lambda)$.

We may choose $|\lambda|$ sufficiently small so that the $(k-1)^{\text {st }}$ image of the critical segment is located inside $S_{\lambda}$. Since the critical circle is mapped $2 n$ times over the critical segment, it follows that the critical circle must be located in exactly one of these disks. The other disk therefore contains no critical points, so the disk that does not contain the critical circle is mapped once over $S_{\lambda}$. The disk with the critical circle inside is mapped $2 n$ times over $S_{\lambda}$, thereby yielding $2 n+1$ preimages of points in $S_{\lambda}$. This gives a contradiction so the preimage of the region $S_{\lambda}$ must be an annulus. Since this annulus contains the critical points, it must surround the origin and hence separate $\mathcal{A}_{\lambda}$ into two open subannuli. Since there are no critical points in these annuli, they are each mapped univalently over $\mathcal{A}_{\lambda}$ by $F_{\lambda}^{k}$. 
Consider the annulus $\mathcal{A}_{\lambda}$ in $\mathcal{O}_{0}(\lambda)$. Its boundary curves $\xi(\lambda)$ and $\nu_{0}(\lambda)$ are each mapped over $\nu_{0}(\lambda) n$ times as a covering by the $k^{\text {th }}$ iterate of the map. Thus $\mathcal{A}_{\lambda}$ is mapped over itself $2 n$ times by $F_{\lambda}^{k}$. Let the preimage of $S_{\lambda}$ in $\mathcal{O}_{0}(\lambda)$ under $F_{\lambda}^{k}$ be denoted $\mathcal{C}_{\lambda}$. By the above proposition, this preimage is an annulus that surrounds the origin. Thus the annulus $\mathcal{A}_{\lambda}$ can be divided into three subannuli: $\mathcal{C}_{\lambda}$ and two others. Let $B_{0}$ be the outer subannulus and $B_{1}$ be the inner subannulus. $\mathcal{C}_{\lambda}$ lies between these two subannuli. See Fig. 5. Under $k$ iterates of $F_{\lambda}, \nu_{0}(\lambda)$ is mapped to itself and both boundary curves of $\mathcal{C}_{\lambda}$ are mapped to $\xi(\lambda)$. Thus $B_{0}$ covers $\mathcal{A}_{\lambda}$ under $F_{\lambda}^{k}$. Similarly, $B_{1}$ is also mapped over $\mathcal{A}_{\lambda}$ by the $k^{\text {th }}$ iterate of $F_{\lambda}$. It follows that there is a preimage of $\mathcal{C}_{\lambda}$ in both $B_{0}$ and $B_{1}$. This construction yields a Cantor set of concentric simple closed curves (or, for short, a Cantor set of circles) in $\mathcal{O}_{0}(\lambda)$ exactly as shown in [McMullen, 1988]. We call this Cantor set of circles $\Lambda_{0}(\lambda)$.

For $j=1, \ldots, k-1$, there is a preimage of this Cantor set of circles in $\mathcal{O}_{j}(\lambda)$. We call this set $\Lambda_{j}(\lambda)$. Note that any point in any of the $\Lambda_{j}(\lambda)$ has orbit that remains for all iterations in $\cup \mathcal{O}_{j}(\lambda)$. Moreover, these points move around the $\mathcal{O}_{j}(\lambda)$ in the exact order that the point $c_{j}$ on the critical orbit moves around $\mathcal{O}_{j}$ under $F_{0}$. Taking preimages of the $\Lambda_{j}(\lambda)$ produces a countable number of Cantor sets of simple closed curves within each of the regions corresponding to a Fatou component of $F_{0}$. We have shown:

Proposition. Let $n \geq 3$. For $z^{n}+\lambda / z^{n}+c$, when $c$ lies at the center of a hyperbolic component in the Multibrot set for $z^{n}+c$ and $|\lambda|$ is sufficiently small, the Julia set contains countably many Cantor sets of simple closed curves.

There is a great deal more structure in the Julia set for functions in this family. In particular, many of the closed curves in the Cantor sets of simple closed curves above come with "decorations." For example, consider the $F_{\lambda}^{k}$ - 
invariant Cantor set of simple closed curves $\Lambda_{0}(\lambda)$ lying in $\mathcal{O}_{0}(\lambda)$. The outer closed curve in this set is the outer boundary of $\mathcal{O}_{0}(\lambda)$, i.e., the simple closed curve $\nu_{0}(\lambda)$. But $\nu_{0}(\lambda)$ is just a piece of $\partial B_{\lambda}$. So we regard the rest of $\partial B_{\lambda}$ as the collection of decorations hanging off $\nu_{0}(\lambda)$.

Now consider the inner circle in this Cantor set of simple closed curves in $\mathcal{O}_{0}(\lambda)$. This is the curve we called $\xi(\lambda)$. This curve is mapped $n$-to-one onto $\nu_{0}(\lambda)$ by $F_{\lambda}^{k}$. Therefore there is a preimage of $\partial B_{\lambda}$ attached to $\xi_{\lambda}$; this preimage is mapped $n$-to-one onto $\partial B_{\lambda}$, i.e., it is the $n$-fold inverted copy of $\partial B_{\lambda}$. These are the decorations attached to this curve; note that they all point inside $\xi_{\lambda}$ and form the boundary of $T_{\lambda}$. Continuing in this manner, each of the two preimages of $\xi(\lambda)$ comes with decorations that are mapped to $\partial T_{\lambda}$. So they are $n^{2}$-inverted copies of $\partial B_{\lambda}$. All of these decorations point into the interior of the first annulus that we removed in the construction of the Cantor set of simple closed curves. Continuing in this fashion, we see that each of the preimages of $\nu_{0}(\lambda)$ also comes with similar decorations atttached. These are the "unburied" curves in $\Lambda_{0}(\lambda)$ so they all come with decorations attached. The uncountably many other simple closed curves in these sets are not so decorated, however, as they do not map to $\xi(\lambda)$. Similar arguments hold for each of the Cantor sets of circles that is a preimage of $\Lambda_{0}(\lambda)$. We have shown:

Proposition. In any preimage of the Cantor set of circles $\Lambda_{0}(\lambda)$,

1. Each of the unburied curves comes with decorations that are preimages of $\partial B_{\lambda}$;

2. Each of the buried components is a simple closed curve without decorations. 


\section{The Rest of the Julia Set}

In the final section, we turn our attention to the dynamical behavior of all the remaining points in the Julia set. In the previous sections, the only repelling periodic points we encountered were located in $\partial B_{\lambda}$ or in one of the $k$ Cantor sets of simple closed curves $\Lambda_{0}(\lambda), \ldots, \Lambda_{k-1}(\lambda)$. In particular, the countably many preimages of $\partial B_{\lambda}$ and the $\Lambda_{j}(\lambda)$ cannot contain any periodic points since these sets eventually map onto $\partial B_{\lambda}$ or the union of the $\Lambda_{j}(\lambda)$ and henceforth remain there. Now it is known that repelling periodic points are dense in the Julia set, so our description of the Julia set up until this point is far from complete. In this section, we prove that the remainder of the Julia set consists of an uncountable collection of point components.

First consider the set of points in $J\left(F_{\lambda}\right)$ that are not in $\partial B_{\lambda}$ or any of its preimages. We shall assign an itinerary to each point in this set. Recall that the disks $\mathcal{O}_{j}$ are the components of the Fatou set of $F_{0}$ that contain $F_{0}^{j}(0)$ for $j=0, \ldots, k-1$, and that the $\mathcal{O}_{j}(\lambda)$ are the corresponding sets for $F_{\lambda}$. The set of bounded components of the Fatou set of $F_{0}$ contains infinitely many other such disks. So for each $j>k-1$, let $\mathcal{O}_{j}$ denote a unique such disk. The way that the $\mathcal{O}_{j}$ are indexed is not important. Let $\mathcal{O}_{j}(\lambda)$ denote the corresponding open disk for $F_{\lambda}$.

We define the itinerary of $z$ by $S(z)=\left(s_{0} s_{1} s_{2} \ldots\right)$ where $s_{j}=\ell$ if and only if $F_{\lambda}^{j}(z) \in \mathcal{O}_{\ell}(\lambda)$. Since, by assumption, the orbit of $z$ never lands in $\partial B_{\lambda}$, there is no ambiguity in this definition. For example, if $z \in \mathcal{O}_{0}(\lambda)$ lies in the $F_{\lambda}^{k}$ invariant Cantor set of circles $\Lambda_{0}(\lambda)$, then $S(z)=(\overline{01 \ldots(k-1)})$. Thus there are infinitely many points that share this particular itinerary. Furthermore, any point that eventually maps to this set must then have itinerary that ends in this repeating sequence.

Now let $\mathcal{J}_{\lambda}$ be the set of points that are not in $\partial B_{\lambda}$ or any of its preim- 
ages and also not in the Cantor sets of simple closed curves $\Lambda_{j}(\lambda)$ or their preimages. We say that $\left(s_{0} s_{1} \ldots\right)$ is an allowable itinerary if this sequence is the itinerary of a point $z \in \mathcal{J}_{\lambda}$. Which sequences are allowable therefore depends on the choice of the indexing of the $\mathcal{O}_{j}(\lambda)$.

Suppose $S(z)=\left(s_{0} s_{1} \ldots\right)$ is an allowable itinerary. If some entry of this itinerary, say $s_{j}$, equals 0 , then either $s_{j+1}=1$ or $s_{j+1} \neq 1$. In the latter case, we call $j$ a departure index. If $j$ is not a departure index, then $s_{j}=0$ is necessarily followed by the string $12 \ldots(k-1) 0$. If $j$ is a departure index, then the orbit of $z$ "deviates" from a similar orbit for $F_{0}$ since it does not move around in the above order. Now each disk $\mathcal{O}_{j}(\lambda)$ must eventually map onto $\mathcal{O}_{0}(\lambda)$ since this is true for $F_{0}$. Therefore there must be infinitely many digits $s_{i}$ for which $s_{i}=0$. Since we have assumed that the orbit of $z$ never lands in the union of the $\Lambda_{j}(\lambda)$, this itinerary cannot end in the repeating sequence $(\overline{01 \ldots(k-1)})$. Therefore it follows that each allowable itinerary must contain infinitely many departure indices.

Proposition. Suppose $\left(s_{0} s_{1} \ldots\right)$ is an allowable itinerary that does not end in the repeating sequence $(\overline{01 \ldots(k-1)})$. Then the set of points with this itinerary is a Cantor set in $J\left(F_{\lambda}\right)$. Moreover, every point in this set is a point component of $J\left(F_{\lambda}\right)$.

Example. Before turning to the proof of this result, we first give an illuminating example. Suppose the given itinerary is $(\overline{0})$. This itinerary is allowable since $F_{\lambda}$ maps $\mathcal{O}_{0}(\lambda)$ over all of the other $\mathcal{O}_{j}(\lambda)$ in $n$-to-one fashion, including $\mathcal{O}_{0}(\lambda)$. Note that each entry in this sequence is a departure index. Since the critical values lie in $\mathcal{O}_{1}$, it follows that there are $n$ subdisks in $\mathcal{O}_{0}(\lambda)$ that are mapped univalently onto $\mathcal{O}_{0}(\lambda)$; these are the points in $\mathbb{C}$ (not just in $J\left(F_{\lambda}\right)$ ) whose itinerary begins 00 . Note that these subdisks lie inside the curve $\xi_{\lambda}$, since the region between $\xi_{\lambda}$ and the outer boundary 
$\nu_{0}(\lambda)$ of $\mathcal{O}_{0}(\lambda)$ is mapped to $\mathcal{O}_{1}(\lambda)$ by $F_{\lambda}$. Hence the union of these $n$ disks is surrounded by infinitely many annuli in $\mathcal{O}_{0}(\lambda)$ that eventually map to the trap door and hence lie in the Fatou set.

Since each of these $n$ disks is mapped univalently onto $\mathcal{O}_{0}(\lambda)$, it follows that each of these subdisks contains preimages of these annuli. Furthermore, each one of these subdisks also contains $n$ smaller subdisks that are mapped univalently onto one of the original $n$ disks. As above, the annuli in these subdisks surround the union of these smaller subdisks. This yields $n^{2}$ smaller disks consisting of points in $\mathbb{C}$ whose itinerary begins 000. Continuing in this fashion, standard arguments from complex dynamics then show that the set of points with itinerary $(\overline{0})$ is a Cantor set of points. Any point in this Cantor set is surrounded by arbitarily small annuli that lie in the Fatou set. Thus each point in this Cantor set is actually a point component of the Julia set.

Proof: The proof of the general case proceeds in essentially the same fashion as the above example, except for the fact that not all of the zeroes in the itinerary correspond to departure indices. Since there are infinitely many zeroes in each allowable itinerary, we may assume without loss of generality that the allowable itinerary begins with a 0 . If this index is a departure index, the itinerary begins with the string $(0 \beta)=\left(0 s_{1} \ldots s_{i-1} 0\right)$ where none of the $s_{j}$ are zero. Then, just as in the example above, the set of points in $\mathbb{C}$ whose itinerary begins with $(0 \beta)$ consists of exactly $n$ disks, and each is mapped univalently onto $\mathcal{O}_{0}(\lambda)$ by $F_{\lambda}^{i}$.

Suppose however that this initial 0 is not a departure index. Then the itinerary begins $0 \alpha=(01 \ldots(k-1) 0)$. As in the previous section, the set of points in $\mathcal{O}_{0}(\lambda)$ whose itinerary begins with this sequence is an annulus, and this annulus is mapped $2 n$-to-one onto $\mathcal{O}_{0}(\lambda)$. If the itinerary begins $(0 \alpha \alpha)$, then the set of points whose itinerary begins in this manner consists of 2 annuli, and each of these annuli is mapped $2 n^{2}$-to-one onto $\mathcal{O}_{0}(\lambda)$. 
Continuing, if the itinerary begins with a total of $m \alpha$ 's after the initial 0 , then the corresponding set consists of $2^{m-1}$ annuli, each of which is mapped $2 n^{m}$-to-one onto $\mathcal{O}_{0}(\lambda)$.

Now suppose that the itinerary begins $(0 \alpha \beta)$. The set of points whose itinerary begins with $(0 \beta)$ consists of $n$ disks in $\mathcal{O}_{0}(\lambda)$. Moreover, none of these disks contains a forward image of a critical point. So if we pull these disks back by the appropriate inverses of $F_{\lambda}$, we find that the set of points whose itinerary begins $(0 \alpha \beta)$ consists of $(2 n) n=2 n^{2}$ disjoint disks in $\mathcal{O}_{0}(\lambda)$, and each of these disks is surrounded by a collecion of annuli lying in the Fatou set. If the itinerary begins $\left(0 \alpha_{m} \beta\right)$, where $\alpha_{m}$ indicates that the itinerary contains $m$ consecutive strings of $\alpha$ 's, then the set of points with this itinerary now consists of $(2 n)^{m} n$ disks, each of which is similarly surrounded by annuli in the Fatou set.

Finally, if we have an itinerary of the form $\left(0 \alpha_{m_{1}} \beta_{1} \alpha_{m_{2}} \beta_{2} \ldots\right)$, concatenating the above constructions shows that the set of points with this itinerary is a nested intersection of disks which converge down to a Cantor set. Moreover, each point in this set is surrounded by arbitrarily small annuli in the Fatou set. This completes the proof. 


\section{References}

Blanchard, P., Devaney, R. L., Look, D. M., Seal, P., and Shapiro, Y. [to appear] "Sierpinski Curve Julia Sets and Singular Perturbations of Complex Polynomials," Ergodic Theory and Dynamical Systems.

Blanchard, P., Devaney, R. L., Garijo Real, A., Marotta, S. M., and Russell, E. D. [to appear] "Rabbits, Basilicas, and Other Julia Sets Wrapped in Sierpinski Carpets."

Devaney, R. L., Josic, K. and Shapiro, Y. [2004] "Singular Perturbations of Quadratic Maps," Intl. J. Bifurcation and Chaos 14, 161-169.

Devaney, R. L., Look, D. M., and Uminsky, D. [2005] "The Escape Trichotomy for Singularly Perturbed Rational Maps," Indiana University Mathematics Journal 54, 1621-1634.

Devaney, R. L. and Look, D. M. [2006] "A Criterion for Sierpinski Curve Julia Sets," Topology Proc. 30, 163-179.

Devaney, R. L. and Marotta, S. M. [2007] "The McMullen Domain: Rings Around the Boundary," Trans. AMS 359, 3251-3273.

Devaney, R. L., Marotta, S. M. [2007] "Evolution of the McMullen Domain for Singularly Perturbed Rational Maps," to appear in Topology Proc.

Douady, A. and Hubbard, J. [1982] "Itération des Polynômes quadratiques complexes," C.R. Acad. Sci. Paris, t.29, Serie I, pp. 123-126.

Mañé, R., Sad, P., and Sullivan, D. [1983] "On the Dynamics of Rational Maps," Ann. Sci. ENS Paris 16, 193-217.

McMullen, C. [1988] "Automorphisms of Rational Maps," Holomorphic Functions and Moduli. Vol. 1. Math. Sci. Res. Inst. Publ. 10. Springer, New York. 
McMullen, C. [1995] "The Classification of Conformal Dynamical Systems," Current Developments in Mathematics. Internat, Press, Cambridge, MA, pp. 323-360.

Milnor, J. [1999] Dynamics in One Complex Variable. Vieweg.

Petersen, C. and Ryd, G. [2000] Convergence of Rational Rays in Parameter Spaces, The Mandelbrot Set: Theme and Variations, London Mathematical Society, Lecture Note Series 274, Cambridge University Press, 161-172.

Whyburn. G. T. [1958] "Topological Characterization of the Sierpinski Curve," Fundamenta Mathematicae 45, 320-324. 